\title{
MODELING AND DIAGNOSIS OF ANALOG CIRCUITS WITH PROBABILISTIC GRAPHICAL MODELS
}

\section{Christian Borgelt}

\author{
School of Computer Science \\ University of Magdeburg, Universitätsplatz 2 \\ D-39106 Magdeburg, Germany \\ e-mail: borgelt@iws.cs.uni-magdeburg.de
}

\author{
Daniela Girimonte, Giuseppe Acciani
}

\author{
Department of Electrotechnics and Electronics \\ Polytechnic of Bari, Via Re David 200 \\ I-70125 Bari, Italy \\ e-mail: \{girimonte,acciani\}@deemail.poliba.it
}

\begin{abstract}
We describe an algorithm to build a graphical model—more precisely: a join tree representation of a Markov networkfor a steady state analog circuit. This model can be used to do probabilistic diagnosis based on manufacturer supplied information about nominal values of electrical components and their tolerances as well as measurements made on the circuit. Faulty components can be identified by looking for high probabilities for values of characteristic magnitudes that deviate considerably from the nominal values.
\end{abstract}

\section{INTRODUCTION}

In the past decades several approaches to the diagnosis of circuits have been developed $[9,10]$. Examples are: the fault dictionary approach, which collects a set of common or relevant faults and associates them with a set of measurements by which they can be identified [2], the model-based diagnosis of digital circuits based on constraint propagation and an assumption-based truth maintenance system (ATMS) [7], and the simulation of a circuit for different predefined faults to generate training data for a classifier, for example, an artificial neural network [1,12]. Especially the diagnosis of digital circuits is well-developed, but it is difficult to extend to analog circuits due to problems like soft faults, (that is, deviations from nominal values) and the non-directional behavior of analog circuits.

Since existing methods for the diagnosis of analog circuits suffer from some drawbacks, like difficulties to take into account tolerances of components and measurements as well as the need for a predefined set of faults, we develop an approach that is based on a probabilistic description of the state of the circuit with the help of a graphical model.

This paper is organized as follows: in Section 2 we briefly review the ideas underlying graphical models with an emphasis on Markov networks. Section 3 explains the iterative proportional fitting method, which is important for the initialization of the probability distributions of the graphical model. In Section 4 we describe our algorithm and illustrate it with a simple example in Section 5. Finally, in Section 6 we draw conclusions and discuss future work.

\section{GRAPHICAL MODELS: MARKOV NETWORKS}

In the last decade graphical models have become one of the most popular tools to structure uncertain knowledge about complex domains $[13,8,3]$ in order to make reasoning in such domains feasible $[11,5]$. Their most prominent representatives are Bayes networks, which are based on directed graphs and conditional distributions, and Markov networks, which are based on undirected graphs and marginal distributions or factor potentials. Here we focus on the latter.

Let $V=\left\{A_{1}, \ldots, A_{n}\right\}$ be a set of (discrete) random variables with respective domains $\operatorname{dom}\left(A_{1}\right), \ldots, \operatorname{dom}\left(A_{n}\right)$. A Markov network is an undirected graph $G=(V, E)$ of these random variables together with a set of functions on the spaces spanned by the variables underlying the maximal cliques $^{1}$ of the graph. The structure of the graph encodes conditional independence statements between (sets of) random variables that hold in the joint probability distribution. This is done by $u$-separation: two (disjoint) sets $X$ and $Y$ of variables are conditionally independent given a third set $Z$ if all paths from a variable in $X$ to a variable in $Y$ contain a variable in $Z$. Conditional independence of $X$ and $Y$ given $Z$, written $X \Perp Y \mid Z$, means that

$$
p_{X Y \mid Z}(x, y \mid z) \equiv p_{X \mid Z}(x \mid z) \cdot p_{Y \mid Z}(y \mid z),
$$

where $x, y$ and $z$ are value vectors from the spaces spanned by the random variables in $X, Y$, and $Z$, respectively. It can be shown [8] that if the graph encodes only correct conditional independences by $u$-separation, then the joint probability distribution $p_{V}$ factorizes according to

$$
p_{V}(v) \equiv \prod_{C \in \mathcal{C}} \phi_{C}(c)
$$

\footnotetext{
${ }^{1}$ A clique is a complete (fully connected) subgraph and it is called maximal if it is not contained in another complete subgraph.
} 
Here the set $\mathcal{C}$ is the set of all sets $C$ of variables underlying the maximal cliques of the graph $G . v$ and $c$ are value vectors over the variables in $V$ and $C$, respectively. The $\phi_{C}$ are the functions on the spaces spanned by the variables in the sets $C \in \mathcal{C}$ already mentioned above. They are called factor potentials [5] and can be defined in different ways from the corresponding marginal probability distributions.

For reasoning purposes a Markov network is often preprocessed into a singly connected structure to avoid update anomalies and incorrect results. The preprocessing consists in triangulating the graph ${ }^{2}$ and turning the resulting hypertree-structured graph into a join tree [5]. In a join tree there is one node for each maximal clique of the graph it is constructed from. In addition, if a variable (node) of the original graph is contained in two nodes of the join tree, it is also contained in all nodes on the path between these nodes in the join tree. A join tree is usually enhanced by so-called node separators on each edge, which contain the intersection of the variables assigned to the connected join tree nodes.

For join trees there exist efficient evidence propagation methods [5] that are based on a message passing scheme, in which the node separators transmit the information between the nodes. In the following we work directly with join trees and neglect that our model is actually a Markov network.

\section{ITERATIVE PROPORTIONAL FITTING}

Iterative proportional fitting (IPF) is a well-known method for adapting the marginal distributions of a given joint probability distribution to desired values [13]. It consists in computing the following sequence of probability distributions:

$$
\begin{aligned}
p_{V}^{(0)}(v) & \equiv p_{V}(v) \\
\forall i=1,2, \ldots: \quad p_{V}^{(i)}(v) & \equiv p_{V}^{(i-1)}(v) \frac{p_{A_{j}}^{*}(a)}{p_{A_{j}}^{(i-1)}(a)}
\end{aligned}
$$

where $j$ is the $((i-1) \bmod |J|+1)$-th element of $J$, the index set that indicates the variables for which marginal distributions are given. $p_{A_{j}}^{*}$ is the desired marginal probability distribution on the domain of the variable $A_{j}, p_{A_{j}}^{(i-1)}$ the corresponding distribution as it can be computed from $p_{V}^{(i-1)}$ by summing over the values of all variables in $V$ except $A_{j}$.

In each step the probability distribution is modified in such a way that it satisfies one given marginal distribution (namely the distribution $p_{A_{j}}^{*}$ ). However, this will, in general, disturb the marginal for a variable $A_{k}$, which has been processed in a previous step. Therefore the adaptation has to be iterated, traversing the set of variables several times.

It can be shown that if there is a solution, IPF converges to a (uniquely determined) probability distribution

\footnotetext{
${ }^{2}$ An undirected graph is called triangulated or chordal if all cycles of length greater than three have a chord, i.e., an edge between two nodes that are nonadjacent in the cycle.
}

that has the desired marginals as well as some other convenient properties $[4,6]$. Convergence may be checked in practice, for instance, by determining the maximal change of a marginal probability: if this maximal change falls below a user-defined threshold, the iteration is terminated.

IPF can easily be extended to probability distributions represented by Markov networks [6]. The idea of this extension is to assign each variable, the marginal distribution of which is to be set, to a maximal clique of the Markov network (or to a node of the join tree it has been turned into), to use steps of iterative proportional fitting to adapt the marginal distributions on the maximal cliques, and to distribute the information added by such an adaptation to the other maximal cliques by standard evidence propagation (preferably carried out by join tree propagation).

\section{CONSTRUCTING THE GRAPHICAL MODEL}

Let $N$ be a time-invariant $n+1$ node, $b$ branch steady state circuit with known topology. Let the nodes be accessible terminals for measurements, so that one of them is taken as a reference (ground) and the node voltages are used to study the circuit. We assume that for each component the electrical law that governs its behavior (e.g. Ohm's law for a resistor), its nominal value(s) and a tolerance provided by the manufacturer are known. We use the following notation:

$$
\begin{array}{lll}
\bar{U}_{i}, & i=1, \ldots, n: & \text { node voltages, } \\
\bar{I}_{j}, & j=1, \ldots, b: & \text { branch currents, } \\
\bar{Z}_{k}=R_{k}+j X_{k}, & k=1, \ldots, b: & \text { branch impedances. }
\end{array}
$$

In order to build a graphical model for the circuit $N$, we have to find partitions of the set of variables $V=\left\{\bar{U}_{1}, \ldots, \bar{U}_{n}\right.$, $\left.\bar{I}_{1}, \ldots, \bar{I}_{b}, \bar{Z}_{1}, \ldots, \bar{Z}_{b}\right\}$ into three disjoint subsets $X_{1}, X_{2}$ and $X_{3}$, such that the variables in $X_{1}$ and $X_{2}$ are conditionally independent given the variables in $X_{3}$. That is, if the values of the variables in $X_{3}$ are fixed, a change of the value of a variable in $X_{1}$ has no influence on the values of the variables in $X_{2}$ and vice versa.

To find such partitions, we consider virtual cross-sections through the circuit (only through wires, not through components). Each of these cross-sections defines a set of variables, namely the voltages of the wires that are cut and the currents flowing through them. Since this set of variables obviously has the property of making the variables on one side of the cross-section independent of those on the other side (and thus satisfies the conditional independence property), we call it a separator set. We select a set of crosssections so that each component is enclosed by two or more cuts or is cut off from the rest of the circuit by a single cut (terminal cross-section). Then the physical law governing a component describes how the variables of its enclosing cross-sections relate to each other. Note that there are usually several ways of selecting the cross-sections. 
Given a set of cross-sections we construct the join tree as follows: the separator sets form the node separators. For each circuit part (containing one component) we create a node containing the union of the separator sets of the bounding cross-sections. In addition, we create a node for each component, comprising the variables needed to describe its behavior, and connect it to the node corresponding to the circuit part the component is in. If the component node contains currents not yet present in the circuit part node, we add these currents to it. The connection is made through an appropriate node separator, containing the intersection of the sets of variables assigned to the connected nodes.

This initial graphical model is simplified in two steps. In the first step, the number of variables is reduced by exploiting trivial Kirchhoff junction equations (like identity of two currents). In the second step, we merge adjacent nodes where the variables in one of them is a subset of the variables in the other. The result is the qualitative part of the graphical model, i.e. the graph structure.

To find the quantitative part (probability distributions), we initialize all node distributions to uniform. Next we enforce Ohm's law as well as Kirchhoff's junction law (wherever applicable). Finally we incorporate the manufacturer supplied information about nominal values and tolerances by iterative proportional fitting (see Section 3). The resulting graphical model can then be used to diagnose the modeled circuit by propagating node voltage measurements, which may be specified as intervals to handle inaccuracies.

From the theory of graphical models it is well known that the computational complexity of operations on a join tree (IPF and evidence propagation) is governed by the size of the node distributions, which depends on the number of variables in a join tree node and the sizes of their domains. If the distributions can be kept small by a proper selection of cross-sections, the computation is very efficient.

\section{A SIMPLE EXAMPLE}

To illustrate our approach we consider the simple resistive circuit shown in Figure 1 , where $n=5, b=7$. It is fed by a voltage supply $U_{1}$, whose internal resistance $R_{1}$ we assume to be zero. The set of (real valued) variables is $V=$ $\left\{U_{1}, \ldots, U_{5}, I_{1}, \ldots, I_{7}, R_{1}, \ldots, R_{7}\right\}$. We select the set of six cross-sections $S_{1}$ to $S_{6}$ that are shown in Figure 2. As an example of the conditional independences referred to above consider the cross-section $S_{3}$ : once we know the voltage of the cut wires $\left(U_{2}\right.$ and $\left.U_{3}\right)$ and the currents through them $\left(I_{2}\right.$ and $I_{4}, I_{4}=I_{2}$ ), all the magnitudes to the left of $S_{3}$ become independent of those to the right of $S_{3}$.

The initial graphical model, as it is constructed from the separator sets, is shown in Figure 3. The node separators (rectangles) are labeled by the cross-sections $S_{1}$ to $S_{6}$ they correspond to. The nodes are drawn with rounded corners

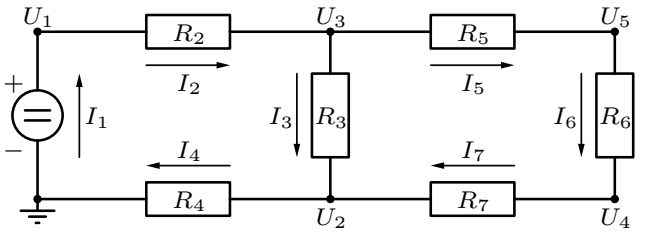

Fig. 1. A simple resistive circuit.

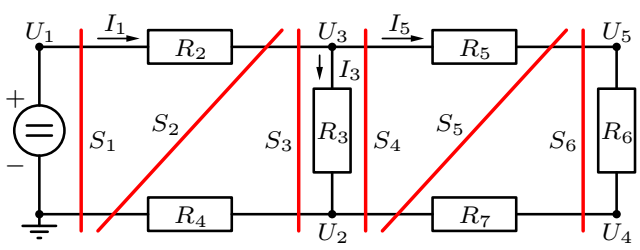

Fig. 2. The resistive circuit with cross-sections.

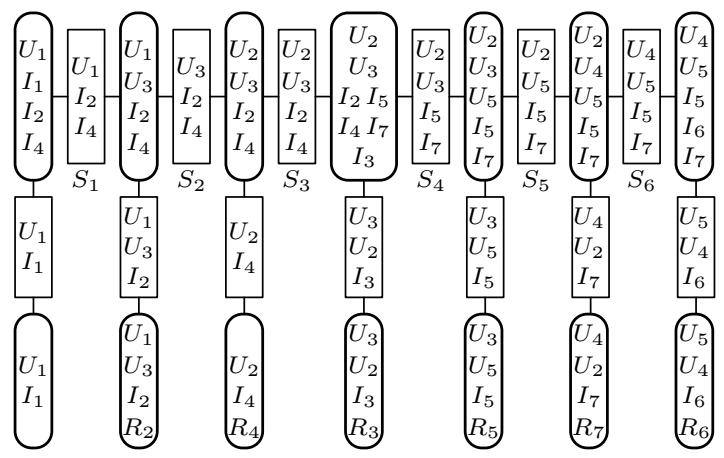

Fig. 3. Initial graphical model for the example circuit.

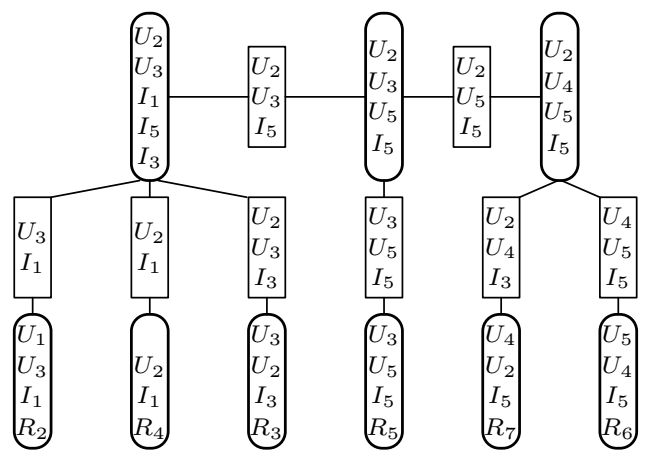

Fig. 4. Simplified graphical model for the example circuit.

and thicker lines. To simplify the network, we exploit $I_{1}=$ $I_{2}=I_{4}$ and $I_{5}=I_{6}=I_{7}$. Furthermore, we merge the four leftmost nodes (two from the top row and two from the bottom row), the third and the fourth nodes on the top row and the two rightmost nodes (the last nodes from the top and bottom row). The result is shown in Figure 4. 


\begin{tabular}{|l|lllll|lllll|}
\hline & \multicolumn{5}{|c|}{$U_{1}=20$} & \multicolumn{4}{c|}{$U_{1}=20 \wedge U_{5}=5$} \\
\hline$R_{2}$ & .11 & .22 & .39 & .19 & .09 & .00 & .04 & .33 & .32 & .31 \\
\hline$R_{3}$ & .09 & .18 & .41 & .21 & .11 & .17 & .23 & .38 & .16 & .07 \\
\hline$R_{4}$ & .12 & .22 & .40 & .18 & .08 & .53 & .29 & .15 & .03 & .00 \\
\hline$R_{5}$ & .11 & .21 & .40 & .19 & .09 & .05 & .15 & .39 & .27 & .15 \\
\hline$R_{6}$ & .11 & .21 & .40 & .19 & .09 & .16 & .25 & .37 & .16 & .07 \\
\hline$R_{7}$ & .11 & .21 & .40 & .19 & .09 & .16 & .25 & .37 & .16 & .07 \\
\hline
\end{tabular}

Table 1. Resistor marginals after propagating the supply voltage $U_{1}=20$ and the measurement $U_{5}=5$.

For our experiments we implemented the described methods for discrete Markov networks in Matlab and C. ${ }^{3}$ We discretized the continuous ranges of values; resistors: 1 to $5 \Omega$ with $1 \Omega$ steps, voltages: 0 to $20 \mathrm{~V}$ with $1 \mathrm{~V}$ steps, currents: 0 to $4 \mathrm{~A}$ with $1 \mathrm{~A}$ steps. For the six resistors we set an initial probability distribution roughly normal and centered at $3 \Omega$, i.e. for $i=2, \ldots, 7: p_{R_{i}}(r)=(0.1,0.2,0.4,0.2,0.1)$.

The initial probability distributions are determined as described in Section 3. With a threshold of $10^{-6}$ the IPF procedure converges after 5 iterations. Next we set the voltage supply to $20 \mathrm{~V}$ and propagate this information using join tree propagation. This changes the marginals of the resistors only slightly as can be seen on the left in Table 1 . Suppose now that we measure the node voltage $U_{5}$ and find it to be $5 \pm 0.5 \mathrm{~V}$ (inaccuracy). Propagating this evidence yields the resistor marginals shown on the right in Table 1 . It can be seen that due to the measurement the distributions for $R_{2}$ and $R_{4}$ change considerably, indicating that at least resistor $R_{4}$ is highly likely to deviate from its nominal value.

\section{CONCLUSIONS AND FUTURE WORK}

We presented a novel method for modeling and diagnosis of analog circuits that exploits probabilistic information about production tolerances of electrical components. It consists of: the construction of a join tree representation of a Markov network from a set of cross-sections of an analog circuit; the IPF procedure for the initialization of the probability distributions; the join tree propagation for the incorporation of (possibly inaccurate) measurements. For our experiments we used a simple example to keep things comprehensible, but the approach is fully general and can be applied to any steady state, AC/DC circuit. Faults like shortcuts or open connections can easily be included by adding them as possible states to the variable(s) describing a component.

In the future we plan to make our method more efficient by exploiting the sparsity of the (discrete) probability distributions (electrical laws rule out a large number of value combinations) and by using parameterized continuous

\footnotetext{
${ }^{3}$ We plan to make the Matlab and $\mathrm{C}$ source files available online at the URL http://fuzzy.cs.uni-magdeburg.de/ ' girimont
}

distributions. Furthermore, we plan to develop a theory of how to select measurements in a diagnosis process. The basic idea is to propagate possible outcomes of measurements through the network, to compute (and to aggregate) the resulting reductions in entropy of the distributions on component values, and finally to select the measurement that leads to the highest expected entropy reduction (similar to [7]).

\section{REFERENCES}

[1] F. Aminian, M. Aminian, and H.W. Collins. Ana$\log$ Fault Diagnosis of Actual Circuits Using Neural Networks. IEEE Trans. Instrumentation and Measurement 51(3):544-550. IEEE Press, Piscataway, NJ, USA 2002

[2] J.W. Bandler and A.E. Salama. Fault Diagnosis of Analog Circuits. Proc. IEEE 73:1279-1325. IEEE Press, Piscataway, NJ, USA 1985

[3] C. Borgelt and R. Kruse. Graphical Models - Methods for Data Analysis and Mining. J. Wiley \& Sons, Chichester, UK 2002

[4] I. Csiszar. I-Divergence Geometry of Probability Distributions and Indirect Observations. Studia Scientiarum Mathematicarum Hungarica 2:299-318. Hungarian Academy of Sciences, Budapest, Hungary 1975

[5] F.V. Jensen. An Introduction to Bayesian Networks. UCL Press, London, UK 1996

[6] R. Jiroušek and S. Pøeuèil. On the Effective Implementation of the Iterative Proportional Fitting Procedure. Computational Statistics and Data Analysis 19:177-189. Int. Statistical Institute, Voorburg, Netherlands 1995

[7] J. de Kleer and B.C. Williams. Diagnosing Multiple Faults. Artificial Intelligence 32(1):97-130. Elsevier Science, New York, NY, USA 1987

[8] S.L. Lauritzen. Graphical Models. Oxford University Press, Oxford, UK 1996

[9] R.-W. Liu, ed. Selected Papers on Analog Fault Diagnosis. IEEE Press, New York, NY, USA 1987

[10] R.-W. Liu. Testing and Diagnosis of Analog Circuits and Systems. Van Nostrand Reinhold, New York, NY, USA 1991

[11] J. Pearl. Probabilistic Reasoning in Intelligent Systems: Networks of Plausible Inference (2nd edition). Morgan Kaufman, San Mateo, CA, USA 1992

[12] R. Spina and S. Upadhyaya. Linear Circuit Fault Diagnosis Using Neuromorphic Analyzers. IEEE Trans. Circuits and Systems II 44(3):188-196. IEEE Press, Piscataway, NJ, USA 1997

[13] J. Whittaker. Graphical Models in Applied Multivariate Statistics. J. Wiley \& Sons, Chichester, UK 1990 